\title{
Overexpression of NCAPH is upregulated and predicts a poor prognosis in prostate cancer
}

\author{
FEILUN CUI, JIANPENG HU, ZHIPENG XU, JIAN TAN and HUAMING TANG \\ Department of Urology, Affiliated People's Hospital of Jiangsu University, Zhenjiang, Jiangsu 212002, P.R. China
}

Received March 6, 2018; Accepted March 18, 2019

DOI: $10.3892 / \mathrm{ol} .2019 .10260$

\begin{abstract}
Prostate cancer $(\mathrm{PCa})$ is one of the most frequently diagnosed types of cancer worldwide. However, there remains a lack of accurate biomarkers to predict the outcome of PCa. Non-SMC condensin I complex subunit H (NCAPH) encodes a regulatory subunit of the non-structural maintenance of chromosomes condensin I complex. The present study aimed to investigate whether NCAPH may be a novel diagnostic marker for PCa by analyzing public datasets, including GSE17951, GSE55945 and a dataset from The Cancer Genome Atlas. The current results, to the best of our knowledge, demonstrated for the first time that NCAPH is significantly upregulated in PCa. Furthermore, it was identified that NCAPH expression is higher in stage T3/T4 and N1 PCa samples compared with stage T2 and N0 PCa samples, respectively. Kaplan-Meier analysis demonstrated that overexpression of NCAPH is associated with poor survival of patients with $\mathrm{PCa}$. Bioinformatics analysis revealed that NCAPH is involved in regulating the PCa cell cycle by interacting with a number of proteins, including non-SMC condensin I complex subunit D2, non-SMC condensin I complex subunit G, structural maintenance of chromosomes 4 , structural maintenance of chromosomes 2, Aurora kinase A, Aurora kinase B, cyclin-dependent kinase 1, H2A histone family member $\mathrm{Z}, \mathrm{POC} 1$ centriolar protein $\mathrm{A}$ and histone cluster $2 \mathrm{H} 2 \mathrm{~A}$ family member C. In summary, the present results suggest NCAPH may be a novel and beneficial diagnostic and therapeutic target in $\mathrm{PCa}$.
\end{abstract}

\section{Introduction}

Condensin serves crucial roles in chromosome-wide gene regulation by controlling sister chromatid cohesion and separation in mitotic and meiotic cell cycles of all proliferative cells $(1,2)$. Structural maintenance of chromosomes (SMC) proteins and three non-SMC subunits, including non-SMC

Correspondence to: Dr Jianpeng Hu, Department of Urology, Affiliated People's Hospital of Jiangsu University, 8 Dianli Road, Zhenjiang, Jiangsu 212002, P.R. China

E-mail: 249056614@qq.com

Key words: prostate cancer, non-SMC condensin I complex subunit $\mathrm{H}$, prognosis, biomarker, cell cycle condensin I complex subunit H (NCAPH), subunit I (NCAPG) and subunit D2 (NCAPD2) form the condensin I complex (3). Previous studies have reported that abnormal expression of the non-SMC condensin I complex is involved in the progression of human cancer (4-6). For example, NCAPG is upregulated and serves as a regulator by regulating hepatocellular cancer cell proliferation and migration $(4,7)$. To the best of our knowledge, no previous study has revealed the prognostic value and the functional roles of the non-SMC condensin I complex in prostate cancer $(\mathrm{PCa})$.

$\mathrm{PCa}$ is one of the most commonly diagnosed malignant cancer types in males worldwide (8), including in China (9). Although several important prognostic indicators, including prostate-specific antigen level, Gleason Score and TNM stage, have widely been used in the diagnosis of PCa $(10,11)$, there remains an urgent requirement to develop novel prognostic biomarkers for PCa. NCAPH is associated with mitotic chromosomes (12) and encodes a regulatory subunit of the non-SMC condensin I complex, which is required for the conversion of interphase chromatin into condensed chromosomes (13). Viera et al (14) identified that NCAPH is localized to the chromosome axis with enrichment at the centromere and telomeric ends. Ryu et al (6) reported that NCAPH is upregulated in melanoma progression. In human colon cancer, Yin et al (5) demonstrated that NCAPH is overexpressed in tumor samples and knockdown of NCAPH significantly inhibited colon cancer cell proliferation in vitro and in vivo. These results suggest NCAPH could be an important diagnostic biomarker and prognostic indicator for cancer. However, the clinical relevance and functional roles of NCAPH in PCa remain unclear.

The aim of the present study was to investigate whether NCAPH may be a novel diagnostic marker and therapeutic target for PCa. The expression pattern of NCAPH was evaluated in PCa tissues and the associations between NCAPH and the clinical features of patients with $\mathrm{PCa}$, including age, pathological stage and overall survival, were evaluated using The Cancer Genome Atlas (TCGA) data. The current study may provide useful information to promote the understanding of the clinical importance of NCAPH in prostate carcinogenesis.

\section{Materials and methods}

Patients and clinicopathological data in TCGA dataset. The detailed expression data of NCAPH in breast cancer 
(BRCA), head and neck squamous cell carcinoma (HNSC), colon adenocarcinoma (COAD), kidney renal clear cell carcinoma (KIRC), liver hepatocellular carcinoma (LIHC), lung squamous cell carcinoma (LUSC), lung adenocarcinoma (LUAD), kidney renal papillary cell carcinoma (KIRP), stomach adenocarcinoma (STAD) and PCa were downloaded from TCGA (https://tcga-data.nci.nih.gov/tcga/) data portal. The RNA expression data (level 3) were generated from a HiSeq 2000 sequencing platform (Illumina, Inc., San Diego, CA, USA) by RNASeqV2 post-processing pipelines and demonstrated as RNA-Seq by Expectation-Maximization normalized count data. Patient clinical data, including age at diagnosis, days to last follow-up, pathological $\mathrm{T}$ stage and pathological $\mathrm{N}$ stage, were retrospectively abstracted from patient records, which were downloaded from cBioPortal (http://www.cbioportal.org/). A total of 112 normal breast samples and 1100 BRCA samples, 44 normal head and neck samples and 522 HNSC samples, 41 normal colon samples and COAD samples, 72 normal kidney renal clear cell samples and 534 KIRC samples, 50 normal liver samples and 373 LIHC samples, 32 kidney renal papillary cell samples and 291 KIRP samples, 35 normal stomach samples and 415 STAD samples, 51 lung squamous cell samples and 501 LUSC samples, and 59 lung samples and 517 LUAD samples were used in the present study. All the patients were staged according to the 2009 TNM classification of the American Joint Committee on Cancer/International Union Against Cancer (15).

The expression levels of NCAPH were downloaded as TCGA data from cBioPortal (http://www.cbioportal. org/) (16). The TCGA PCa dataset was analyzed to evaluate the expression pattern of NCAPH in PCa samples. A total of 38 normal and $497 \mathrm{PCa}$ samples were included in this dataset. Normal prostate tissue samples analyzed from the TGCA cohort were obtained from the marginal area of tumours derived from the same patient and were therefore paired samples. The median age of the patients in the TCGA PCa dataset was 62 years (range, 41-78 years). NCAPH protein expression levels in the PCa tissues and normal prostate tissues were reviewed using the Human Protein Atlas (http://www.proteinatlas.org/) (17).

Microarray data. Three public datasets GSE21034 (18), GSE17951 (19) and GSE55945 (20) were download from the Gene Expression Omnibus (GEO) database (www.ncbi.nlm. nih.gov/geo/). GSE21034 (Taylor dataset) was reported by Taylor et al (18) and includes 370 PCa tissue samples and cell line samples. The GSE17951 dataset was reported by Jia et al (19) and contains 70 normal prostate and $67 \mathrm{PCa}$ samples. The GSE55945 dataset was reported by Arredouani et al (20) and contains eight normal prostate and $13 \mathrm{PCa}$ samples. Unfortunately, the clinicopathological features of the patients were not available in the three GEO datasets. Genes with a fold-change $\geq 2$ and $\mathrm{P}<0.05$ were considered to be significantly differentially expressed.

Gene ontology (GO) and Kyoto Encyclopedia of Genes and Genomes (KEGG) pathway analysis. To identify functions of NCAPH in PCa, GO functional enrichment analysis was performed for three functional ontologies: Biological process, cellular component and molecular function. KEGG pathway enrichment analysis was also performed to identify pathways enriched in PCa using the Database for Annotation, Visualization and Integrated Discovery (DAVID; https://david. ncifcrf.gov/home.jsp) (21). The P-value was calculated by hypergeometric distribution, and a pathway with $\mathrm{P}<0.05$ was considered as significant.

Protein-protein interaction (PPI) network construction. Using a NCAPH interacting proteins dataset which was download from NCBI (https://www.ncbi.nlm.nih.gov/gene/23397), a total of 51 candidate proteins were identified. The Search Tool for the Retrieval of Interacting Genes/Proteins (STRING; string-db.org/) database is a pre-computed global resource for the investigation and analysis of associations between proteins (22). STRING was used to analyze the interactions between NCAPH-associated proteins, which were visualized with PPI networks. In the present study, only experimentally validated interactions with a combined score $\geq 0.4$ were selected as significant. PPI networks were constructed using Cytoscape 3.6.1 software (http://www.cytoscape.org/) (23).

Statistical analysis. SPSS 17.0 software (SPSS, Inc., Chicago, IL, USA) was used for all statistical analysis. Pearson's $\chi^{2}$ test and Fisher's exact test were used to analyze the associations of NCAPH expression with clinicopathological characteristics. Paired and independent Student's t-tests were used to compare continuous variables in two groups. Receiver operating curve (ROC) analysis was performed for evaluation of the specificity and sensitivity of NCAPH expression levels for discriminating patients with PCa from normal patients. Associations between continuous variables and categorical variables were evaluated using Mann Whitney U tests for two groups. Kaplan-Meier and Cox regression analyses were utilized to assess the associations between NCAPH and overall survival, as well as the prognosis of patients with PCa. A log-rank test was used to compare differences in survival times. All tests were two-sided and $\mathrm{P}<0.05$ was considered to indicate a statistically significant difference. All numerical data are presented as the mean \pm standard deviation of at least three independent experiments.

\section{Results}

NCAPH is upregulated in PCa. The expression patterns of $\mathrm{NCAPH}$ in ten types of human cancer types, including BRCA, HNSC, COAD, KIRC, LIHC, LUSC, LUAD, KIRP, STAD and PCa, were analyzed. It was identified that NCAPH was significantly overexpressed in cancer tissues compared with normal samples (Figs. 1A-I and 2A), which suggests that NCAPH serves as an oncogene in human cancer. The present study then focused on the clinical roles of NCAPH in PCa.

As presented in Fig. 2A and B and Table I, TCGA analysis demonstrated that NCAPH was upregulated in PCa tissues compared with non-tumor tissues. In addition, it was identified that NCAPH could serve as a specific and sensitive biomarker for PCa (area under the curve, 0.769; 95\% confidence interval, 0.701-0.836; $\mathrm{P}<0.0001$; Fig. $2 \mathrm{C}$ ). To further validate this result, two independent public datasets GSE17951 (24) and GSE55945 (20) were analyzed. NCAPH was revealed to be overexpressed in PCa compared with normal samples in the GSE17951 dataset (P<0.001; Fig. 2D) and the GSE55945 dataset (P<0.05; Fig. 2E). 
A

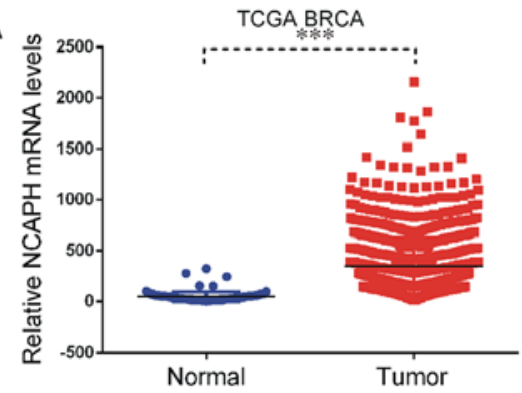

D

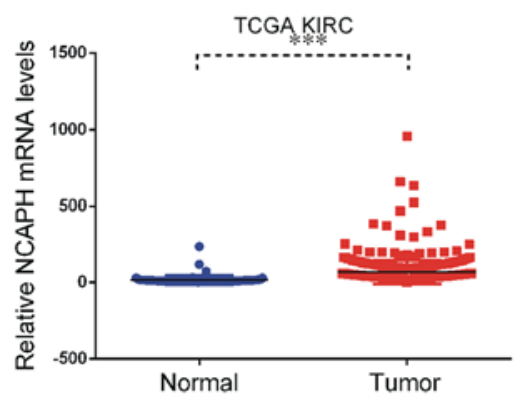

G

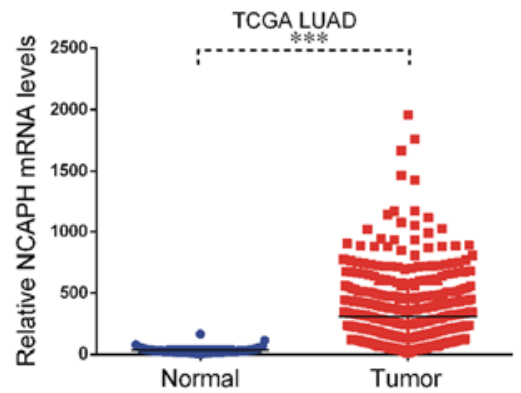

B

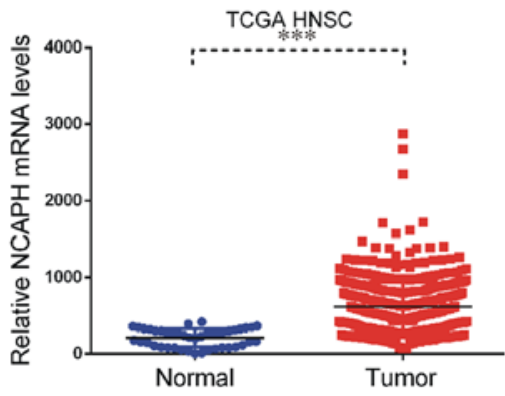

E

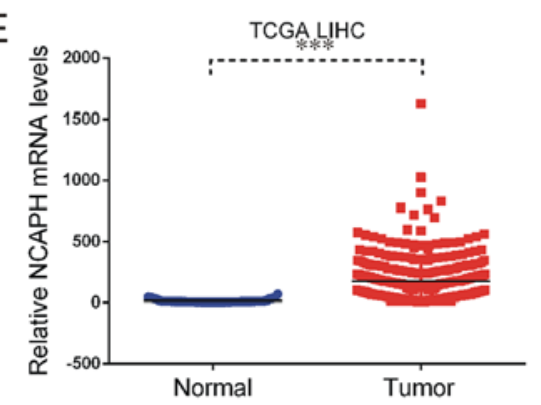

$\mathrm{H}$

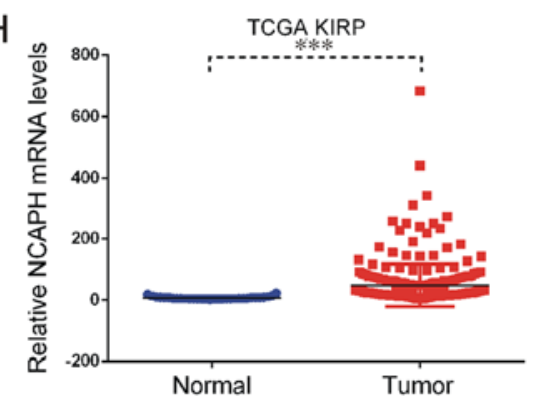

C

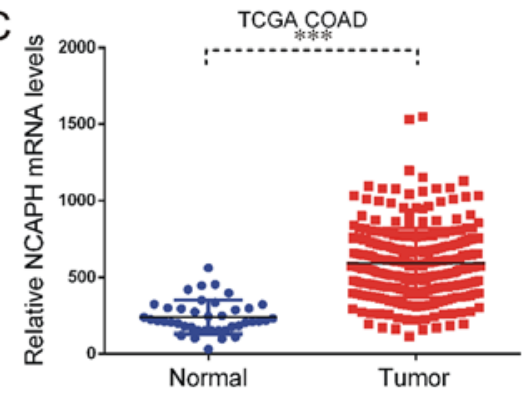

F

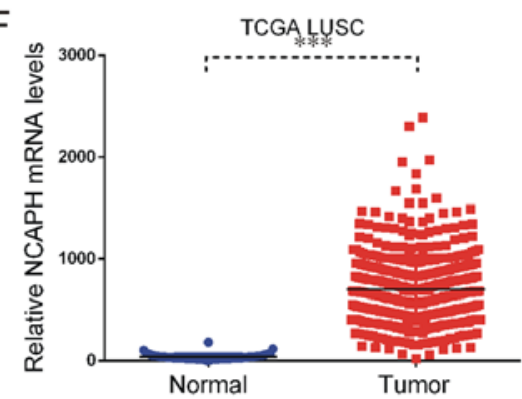

I

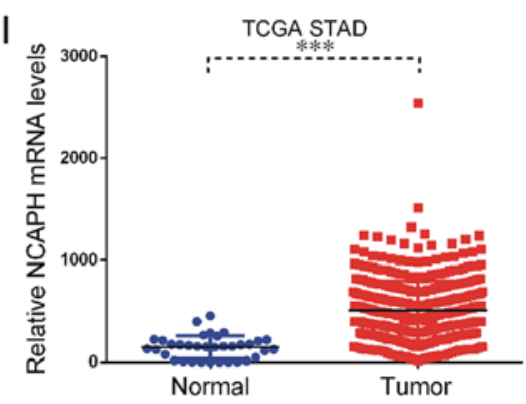

Figure 1. NCAPH is upregulated in human cancer types. TCGA data analysis revealed that NCAPH expression is significantly upregulated in (A) BRCA, (B) HNSC, (C) COAD, (D) KIRC, (E) LIHC, (F) LUSC, (G) LUAD, (H) KIRP and (I) STAD samples compared with matched normal samples. ${ }^{* * *}$ P $<0.001$. $\mathrm{NCAPH}$, non-SMC condensin I complex subunit H; BRCA, breast cancer; HNSC, head and neck squamous cell carcinoma; COAD, colon adenocarcinoma; KIRC, kidney renal clear cell carcinoma; LIHC, liver hepatocellular carcinoma; LUSC, lung squamous cell carcinoma; LUAD, lung adenocarcinoma; KIRP, kidney renal papillary cell carcinoma; STAD, stomach adenocarcinoma; TCGA, The Cancer Genome Atlas.

NCAPH is associated with pathological stage in PCa. The possible prognostic values of NCAPH were then evaluated using TCGA RNA-seq data. Associations between NCAPH mRNA expression and the clinicopathological characteristics of PCa are summarized in Table II. Positive expression of NCAPH was associated with age (Fig. 3A; Table II) and pathological stage (Fig. 3B and C; Table II). As presented in Fig. 3, NCAPH expression was significantly upregulated in T3/T4 PCa samples compared with T2 samples (Fig. 3B) and in N1 stage PCa samples compared with N0 samples (Fig. 3C). However, univariate analysis indicated that age, $\mathrm{T}$ stage and $\mathrm{N}$ stage were not associated with overall survival in PCa (Table III). These results indicate that NCAPH is associated with the pathological stage of PCa.

Upregulation of NCAPH predicts a poor prognosis in $\mathrm{PCa}$. Furthermore, Kaplan-Meier analysis was performed to determine whether NCAPH expression is significantly associated with overall survival of patients with PCa. Two independent public datasets were analyzed, including the Taylor dataset (18) and TCGA dataset. The median NCAPH mRNA expression level in PCa tissues was used as the cut-off point to divide all cases into NCAPH-high (Taylor dataset, $\mathrm{n}=71$; TCGA dataset, $\mathrm{n}=248$ ) and NCAPH-low (Taylor dataset, $\mathrm{n}=71$; TCGA dataset, $\mathrm{n}=247$ ) groups. The samples with NCAPH expression higher than median value were identified as NCAPH-high, and the samples with NCAPH expression lower than median value were identified as NCAPH-low. In TCGA analysis, the overall survival rate was significantly higher in the NCAPH-low group compared with the NCAPH-high group $(\mathrm{P}=0.0012$; Fig. 4A). In addition, similar results were observed with the Taylor dataset ( $\mathrm{P}=0.0099$; Fig. 4B). These results indicate that NCAPH expression could serve as a biomarker to predict the outcome of PCa.

Construction of NCAPH mediated PPI networks in PCa. The molecular functions of NCAPH in PCa remain largely unclear. Therefore, the current study performed PPI network analysis to reveal the potential roles of NCAPH. Using a NCAPH interacting proteins dataset which was download from NCBI (https://www.ncbi.nlm.nih.gov/gene/23397), a total of 51 candidate proteins were identifed. By applying STRING analysis, 10 of the 51 proteins [NCAPG, NCAPD2, structural maintenance of chromosomes 4 (SMC4), structural maintenance of chromosomes 2 (SMC2), Aurora kinase A (AURKA), Aurora kinase B (AURKB), cyclin-dependent kinase 1 (CDK1), H2A 
Table I. Expression levels of NCAPH in paired prostate cancer samples by using The Cancer Genome Atlas database.

\begin{tabular}{|c|c|c|c|c|c|c|c|}
\hline ID & $\begin{array}{c}\text { Gene } \\
\text { symbol }\end{array}$ & $\mathrm{FC}$ & P-value & $\begin{array}{c}\text { Total } \\
\text { samples }\end{array}$ & $\begin{array}{c}\text { Samples } \\
\text { unchanged }\end{array}$ & $\begin{array}{c}\text { Samples } \\
\text { upregulated }\end{array}$ & $\begin{array}{c}\text { Samples } \\
\text { downregulated }\end{array}$ \\
\hline 23397 & NCAPH & 3.522 & $2.59 \times 10^{-17}$ & 38 & 10 & 27 & 1 \\
\hline
\end{tabular}

NCAPH, non-SMC condensin I complex subunit H; FC, fold-change.

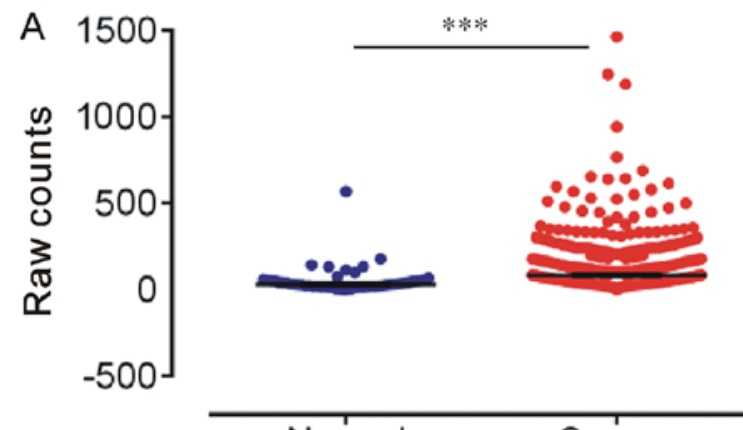

Normal Cancer

C

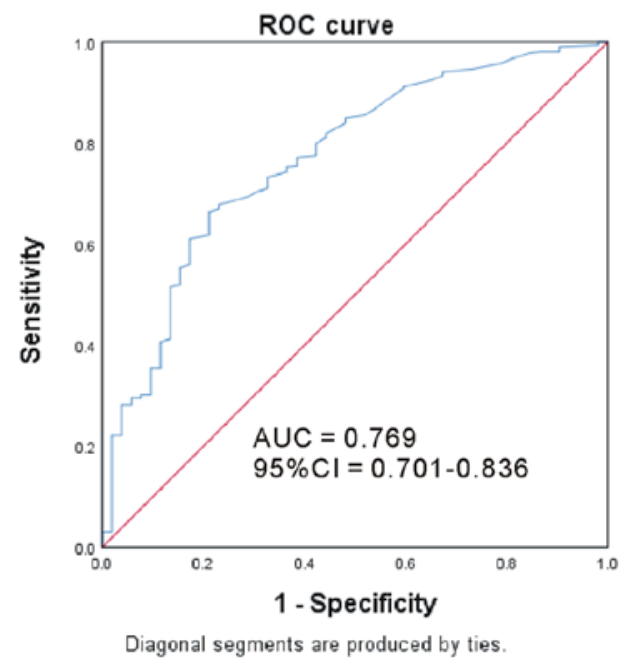

E

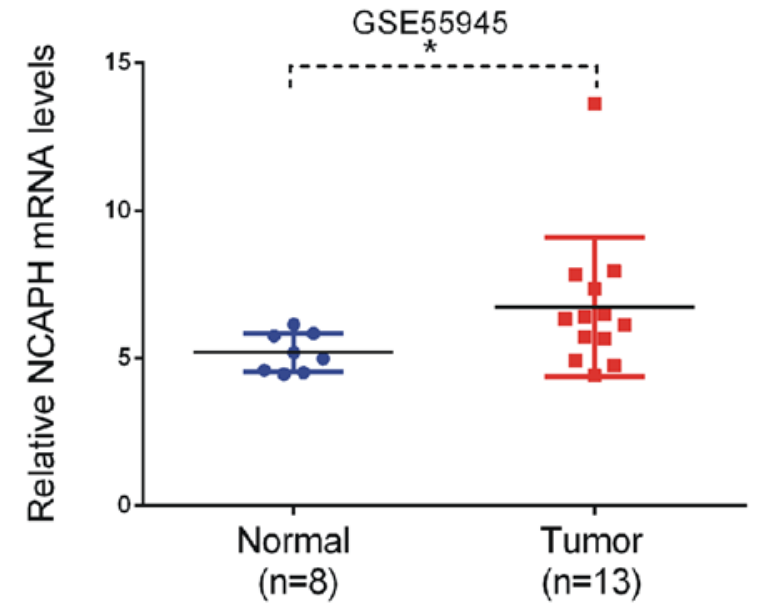

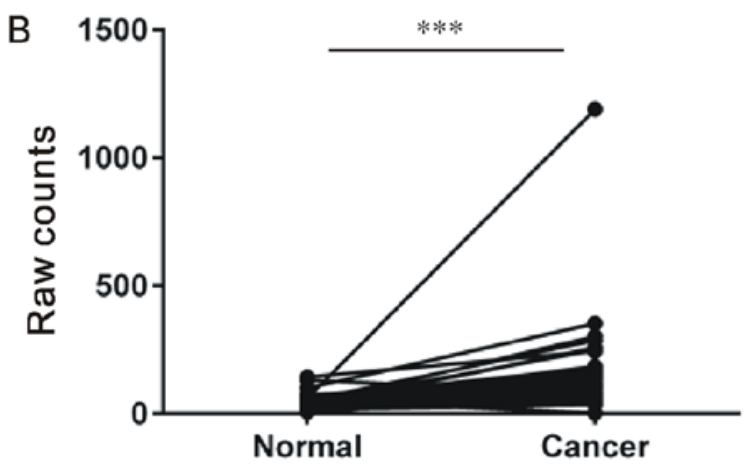

D

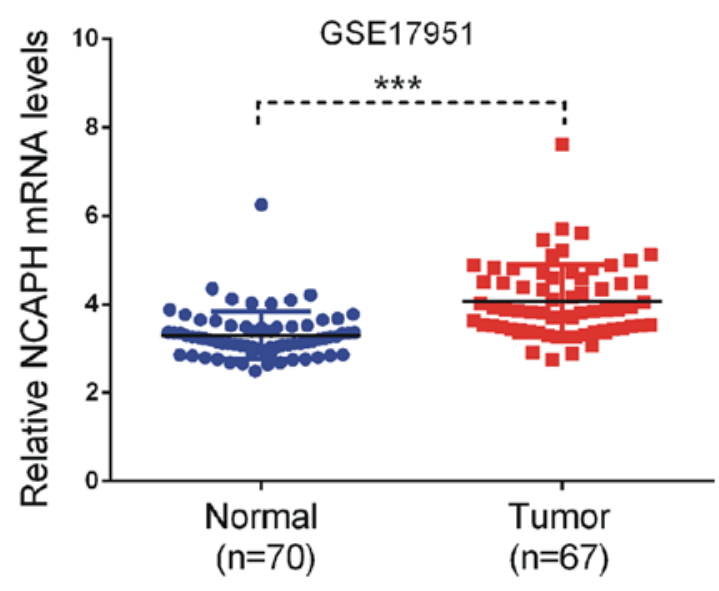

Figure 2. NCAPH expression level is upregulated in prostate cancer. (A) The Cancer Genome Atlas data analysis revealed that NCAPH expression levels were significantly upregulated in prostate cancer samples compared with normal prostate tissues. (B) The Cancer Genome Atlas data analysis revealed that NCAPH expression levels were significantly upregulated in 38 prostate cancer samples compared with match normal tissues. (C) ROC analysis revealed NCAPH could serve as a specific and sensitive biomarker in prostate cancer. (D) GSE17951 dataset analysis demonstrated that NCAPH expression levels were significantly higher in prostate tumor samples compared with normal prostate tissues (E) GSE55945 data analysis revealed NCAPH expression levels were significantly upregulated in prostate cancer samples compared with normal prostate tissues. ${ }^{*} \mathrm{P}<0.05,{ }^{* * *} \mathrm{P}<0.001$. NCAPH, non-SMC condensin I complex subunit $\mathrm{H}$; ROC, receiver operating curve; $\mathrm{AUC}$, area under the curve; $\mathrm{CI}$, confidence interval. 
Table II. Associations of NCAPH expression with clinicopathological characteristics of patients with prostate cancer in The Cancer Genome Atlas dataset.

\begin{tabular}{|c|c|c|c|c|}
\hline \multirow[b]{2}{*}{ Characteristic } & \multicolumn{2}{|c|}{$\begin{array}{c}\text { NCAPH } \\
\text { expression level }\end{array}$} & \multirow[b]{2}{*}{ Total } & \multirow[b]{2}{*}{ P-value } \\
\hline & High & Low & & \\
\hline Age, years & & & & 0.022 \\
\hline$\leq 62$ & 147 & 121 & 268 & \\
\hline$>62$ & 102 & 127 & 229 & \\
\hline Total & 249 & 248 & 497 & \\
\hline T stage & & & & $<0.001$ \\
\hline $\mathrm{T} 2$ & 122 & 65 & 187 & \\
\hline $\mathrm{T} 3 / 4$ & 123 & 180 & 303 & \\
\hline Total & 244 & 246 & 490 & \\
\hline N stage & & & & 0.009 \\
\hline NO & 174 & 171 & 345 & \\
\hline N1 & 27 & 52 & 79 & \\
\hline Total & 201 & 223 & 424 & \\
\hline
\end{tabular}

NCAPH, non-SMC condensin I complex subunit H.
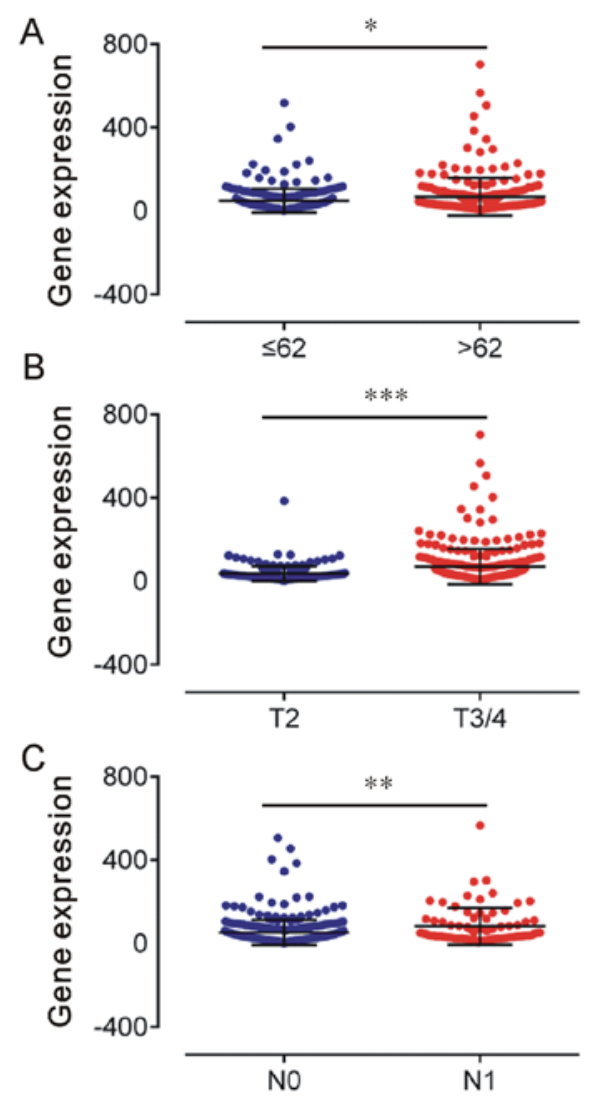

Figure 3. NCAPH expression is upregulated in high pathological T/N stage prostate cancer. (A) NCAPH expression was upregulated in patients with prostate cancer with an age $>62$ years compared with patients $\leq 62$ years old. (B) NCAPH expression was significantly upregulated in T3/4 stage prostate cancer samples compared with $\mathrm{T} 2$ stage prostate cancer samples. (C) $\mathrm{NCAPH}$ expression was significantly upregulated in N1 stage prostate cancer samples compared with N0 stage prostate cancer samples. " $\mathrm{P}<0.05$, ${ }^{* *} \mathrm{P}<0.01,{ }^{* * * *} \mathrm{P}<0.001$. NCAPH, non-SMC condensin I complex subunit $\mathrm{H}$.
Table III. Univariate analysis of overall survival for patients with prostate cancer.

\begin{tabular}{lccc}
\hline Variable & $\chi^{2}$ & df & Log-rank P-value \\
\hline Age & 0.741 & 1 & 0.389 \\
N stage & 3.101 & 1 & 0.078 \\
T stage & 2.068 & 1 & 0.150 \\
\hline
\end{tabular}

df, degree freedom.
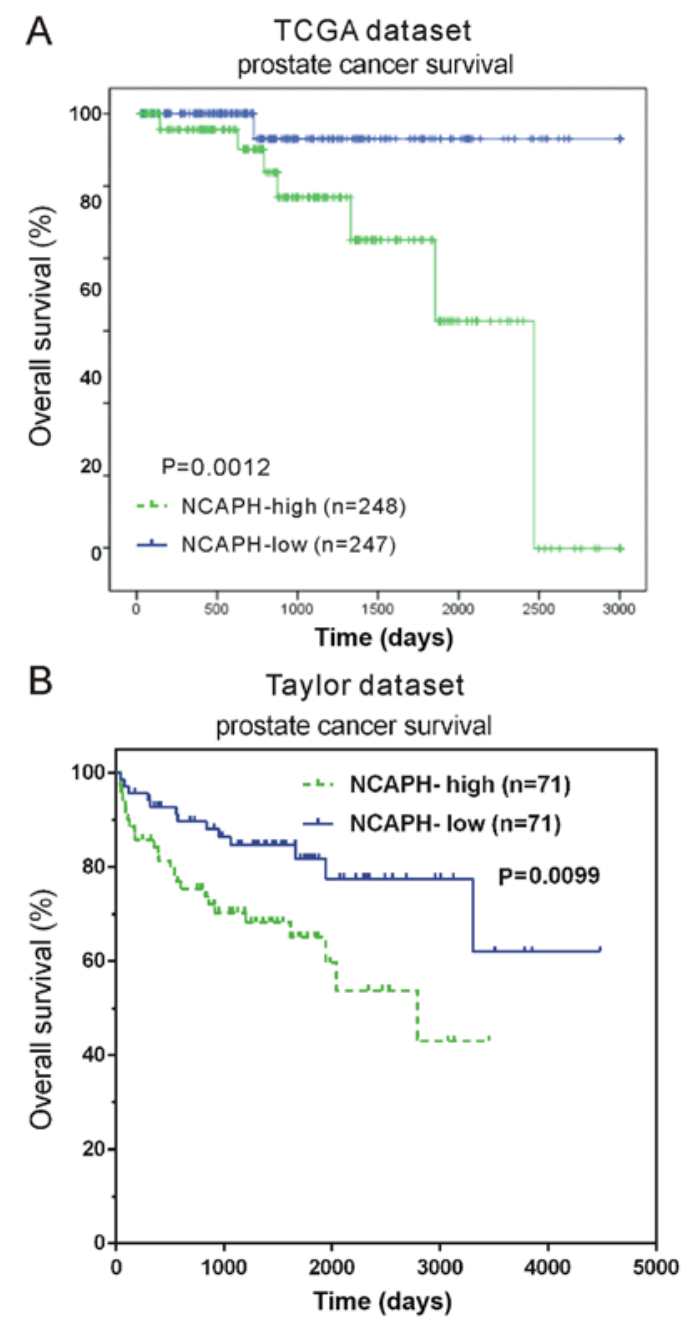

Figure 4. Kaplan-Meier survival analysis of the association of overall survival rate with NCAPH expression in prostate cancer. Compared with patients with high NCAPH expression, the overall survival rates were higher for patients with low NCAPH expression in the (A) TCGA and (B) Taylor datasets. NCAPH, non-SMC condensin I complex subunit H; TCGA, The Cancer Genome Atlas

histone family member $\mathrm{Z}$ (H2AFZ), $\mathrm{POC} 1$ centriolar protein A (POC1A) and histone cluster $2 \mathrm{H} 2 \mathrm{~A}$ family member C (HIST2H2AC)] were identified to interact with NCAPH with a combined score $\geq 0.4$ and selected as seed genes.

PPI networks were then generated with the NCAPH interacting seed genes by applying STRING analysis. A total of 417 single nodes and 10,071 edges were included in the NCAPH-mediated PPI network. According to a previous study 
A
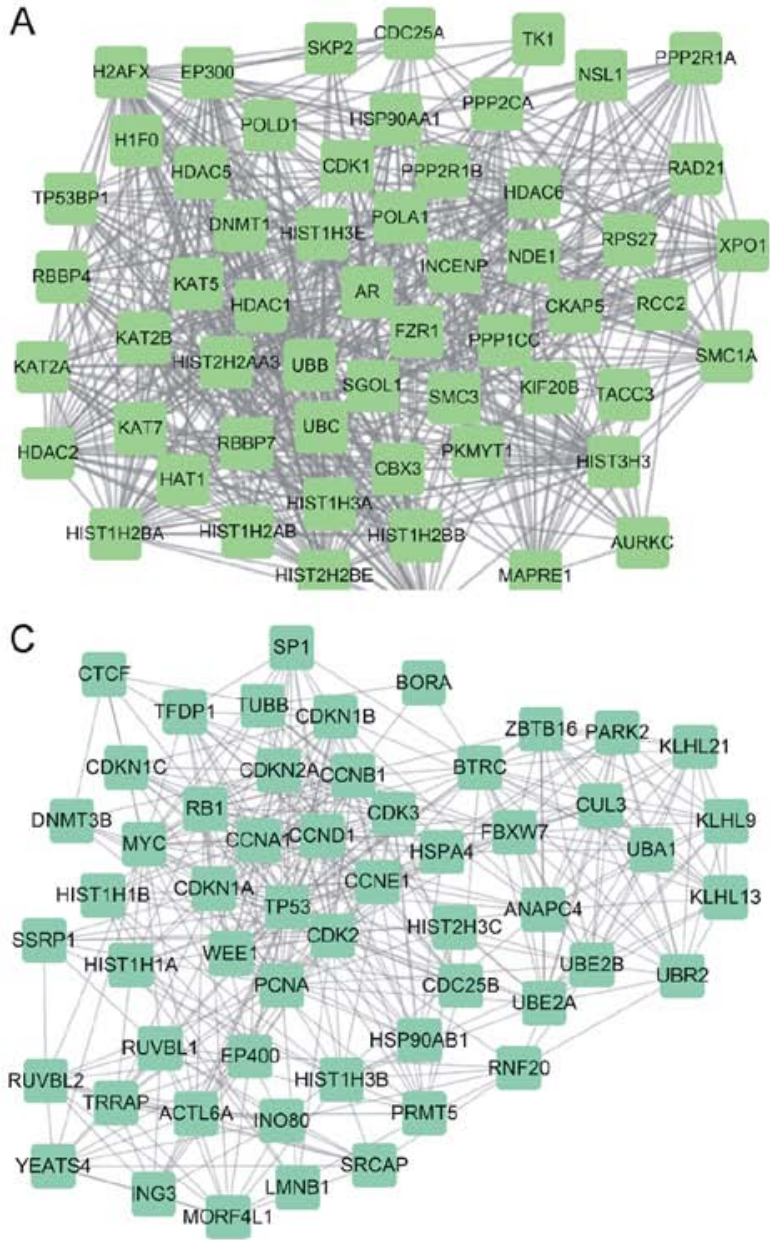

$\mathrm{E}$

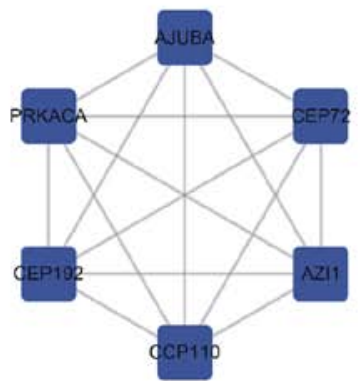

B

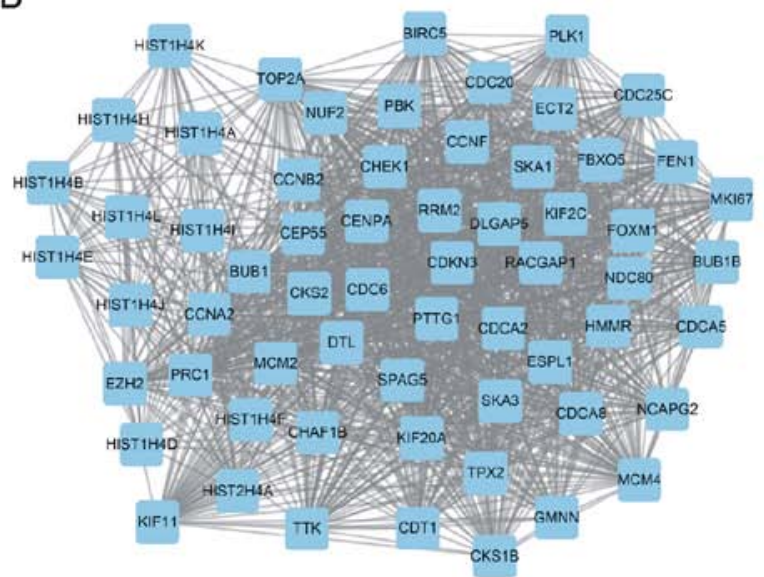

D

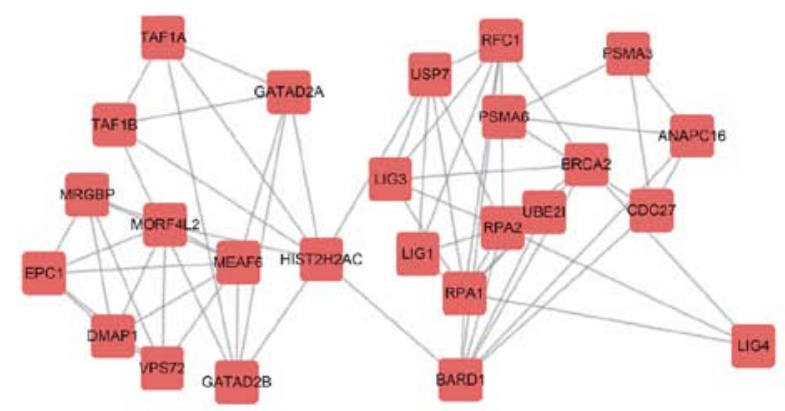

$\mathrm{F}$

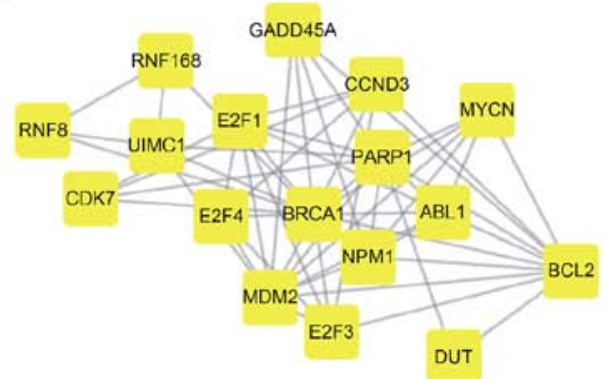

Figure 5. Construction of NCAPH-mediated protein-protein interaction networks in prostate cancer. A total of six hub NCAPH-mediated protein-protein interaction networks were constructed in prostate cancer. (A) Hub-network 1 contains 62 nodes and 1,325 edges. (B) Hub-network 2 includes 57 nodes and 627 edges. (C) Hub-network 3 consists of 55 nodes and 418 edges. (D) Hub-network 4 contains 17 nodes and 57 edges, (E) Hub-network 5 includes 6 nodes and 15 edges. (F) Hub-network 6 consists of 25 nodes and 65 edges. NCAPH, non-SMC condensin I complex subunit H.

by Zhang et al (25), the 'K value' of the PPI network was calculated to identify the hub networks in PCa. Finally, a total of six hub networks were revealed. As presented in Fig. 5, hub-network 1 contained 62 nodes and 1,325 edges (Fig. 5A), hub-network 2 consisted of 57 nodes and 627 edges (Fig. 5B), hub-network 3 contained 55 nodes and 418 edges (Fig. 5C), hub-network 4 consisted of 17 nodes and 57 edges (Fig. 5D), hub-network 5 included six nodes and 15 edges (Fig. 5E) and hub-network 6 was composed of 25 nodes and 65 edges (Fig. 5F).

Enrichment analysis of NCAPH in PCa. To investigate the biological characteristics of NCAPH in $\mathrm{PCa}$, enrichment analysis based on the GO annotation system was performed.
A total of 417 genes in the NCAPH-mediated PPI network were classified according to GO terms using DAVID. GO analysis demonstrated that NCAPH is predominantly involved in regulating $\mathrm{PCa}$ cell cycle progression and cell proliferation (Fig. 6A). A series of associated biological process terms, including 'Cell division', 'Mitotic nuclear division', 'G2/M transition of mitotic cell cycle', 'G1/S transition of mitotic cell cycle', 'Nucleosome assembly', 'DNA repair', 'Negative regulation of gene expression, epigenetic', 'DNA replication' and 'Sister chromatid cohesion' were enriched in GO analysis. Molecular function analysis demonstrated NCAPH is associated with 'Protein binding', 'Histone binding' and 'Chromatin binding' (Fig. 6B). 
A

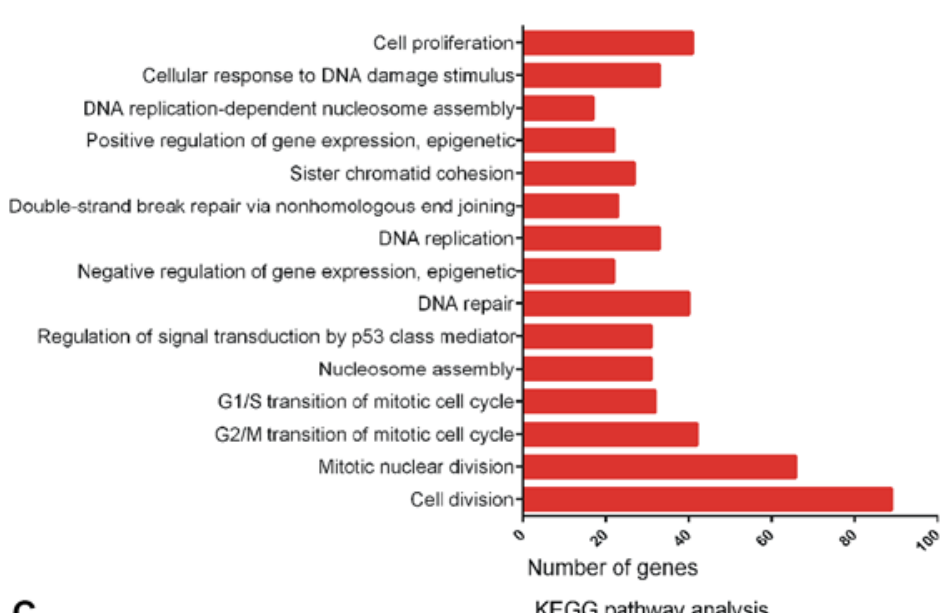

C

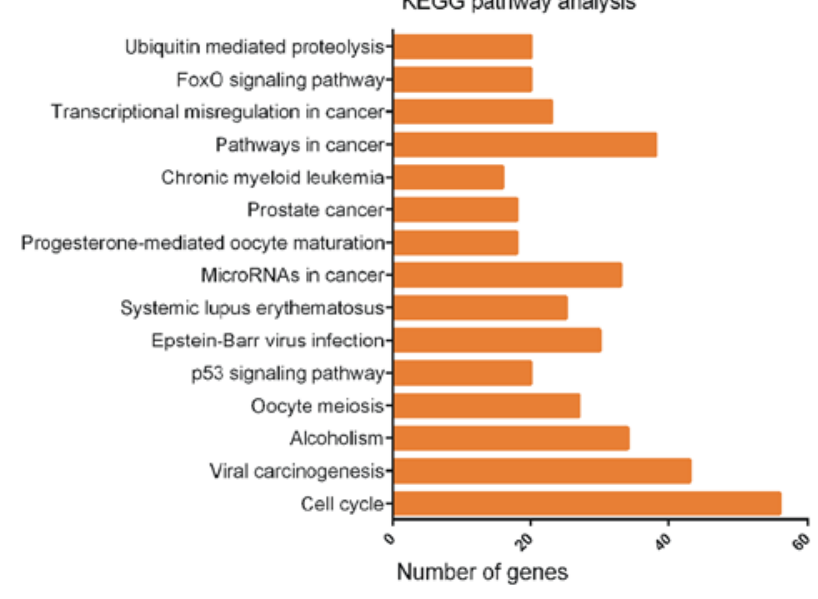

B

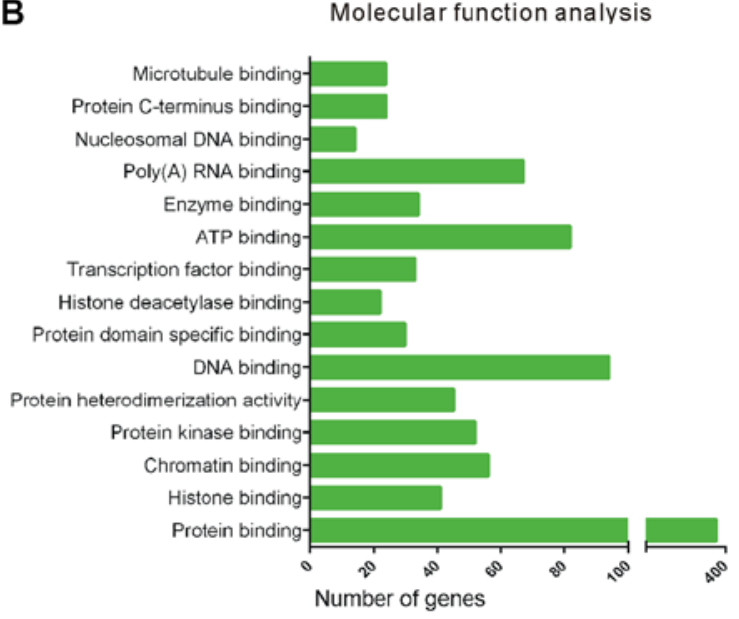

Figure 6. GO and KEGG pathway analysis of NCAPH. (A) Biological processes, (B) molecular function and (C) KEGG pathway analysis of NCAPH based on the protein-protein interaction network analysis using the Database for Annotation, Visualization and Integrated Discovery. NCAPH, non-SMC condensin I complex subunit H; GO, gene ontology; KEGG, Kyoto Encyclopedia of Genes and Genomes; FoxO, Forkhead box O.

Furthermore, KEGG analysis revealed NCAPH is significantly associated with 'Cell cycle', 'Viral carcinogenesis', 'Alcoholism', 'Oocyte meiosis', 'p53 signaling pathway', 'Epstein-Barr virus infection', 'Systemic lupus erythematosus', 'MicroRNAs in cancer', 'Progesterone-mediated oocyte maturation' and 'Prostate cancer' (Fig. 6C).

\section{Discussion}

$\mathrm{PCa}$ is one of the most frequently diagnosed cancer types worldwide $(8,26)$. However, to the best of our knowledge, there remains a lack of accurate biomarkers to predict the outcome of PCa. The present study identified that NCAPH is significantly upregulated in tumor samples and could serve as a biomarker for PCa (14). To reveal the potential roles of NCAPH in PCa, NCAPH-mediated PPI networks were constructed. Bioinformatics analysis was also performed to predict the potential functional roles of NCAPH in PCa.

NCAPH, NCAPG and NCAPD2 form the non-SMC condensin I complex, which serves crucial roles in chromosome-wide gene regulation in mitotic and meiotic cell cycles by controlling chromosome organization (1). Previous studies have indicated the important roles of the condensin I complex in human cancer (4-6). NCAPH has been reported to be upregulated in melanoma and colon cancer $(5,6)$. The present study demonstrated that NCAPH is upregulated in PCa samples by analyzing three public datasets, including TCGA data, GSE17951 and GSE55945. In addition, it was identified that NCAPH is upregulated in T3/T4 PCa samples compared with $\mathrm{T} 2$ samples and upregulated in N1 stage PCa samples compared with N0 samples. Furthermore, Kaplan-Meier analysis revealed that patients with PCa with low NCAPH expression exhibited longer overall survival times compared with patients with high NCAPH expression. The present analysis was similar to a previous study regarding human colon cancer (5). Yin et al (5) also observed that NCAPH expression is higher in colon cancer tissues compared with adjacent normal tissues. However, the higher NCAPH expression in colon cancer was associated with an improved prognosis and longer survival time. One limitation of the current study should be noted; the detailed clinical information of the patients in the four public datasets was not completed. To the best of our knowledge, the present study reported for the first time that NCAPH is associated with the prognosis of $\mathrm{PCa}$.

A number of studies have demonstrated that oncogenes or tumor-suppressive genes cooperate in interconnected pathways and molecular networks at different levels to regulate cancer progression $(27,28)$. The present study constructed a NCAPH-mediated PPI network to reveal the 
associations of NCAPH in PCa, which included 417 single nodes and 10,071 edges. Abnormal cell cycle progression is a hallmark of human cancer $(29,30)$. Therefore, targeting cell-cycle checkpoints could provide novel strategies for cancer treatment. Notably, GO and KEGG analysis revealed that NCAPH predominantly participates in regulating the PCa cell cycle and proliferation by affecting a series of associated biological processes, including cell division, mitotic nuclear division, G2/M transition of mitotic cell cycle and G1/S transition of mitotic cell cycle. Of note, Yin et al (5) also demonstrated that knockdown of NCAPH could induce cell cycle arrest at the G2/M phase. The present study together with other previous studies indicate that NCAPH-mediated cell cycle regulation is a mechanism involved in PCa proliferation. These results suggest NCAPH may be a therapeutic target for $\mathrm{PCa}$.

In conclusion, the present study demonstrated for the first time that NCAPH was significantly upregulated in PCa. Furthermore, Kaplan-Meier analysis revealed that overexpression of NCAPH is associated with poor survival of patients with $\mathrm{PCa}$. Bioinformatics analysis revealed NCAPH is involved in regulating $\mathrm{PCa}$ cell cycle by interacting with ten proteins, including NCAPD2, NCAPG, SMC4, SMC2, AURKA, AURKB, CDK1, H2AFZ, POC1A, HIST2H2AC. In summary, the current results suggest NCAPH may be a potential beneficial and novel diagnostic and therapeutic marker in PCa.

\section{Acknowledgements}

Not applicable.

\section{Funding}

The present study was supported by the Social Development Plan of Jiangsu Province-Standardization of Key Disease Diagnosis and Treatment Projects (grant no. BE2016715) and the Jiangsu Province Youth Medical Key Talent Program (grant no. QNRC2016457).

\section{Availability of data and materials}

The datasets used and/or analyzed during the current study are available from the corresponding author on reasonable request.

\section{Authors' contributions}

FC and $\mathrm{JH}$ were responsible for the study conception and design. FC, JH and HT developed the methodology. FC, JT and $\mathrm{ZX}$ analyzed and interpreted the data. FC and $\mathrm{JH}$ wrote, reviewed and revised the manuscript.

\section{Ethics approval and consent to participate}

Not applicable.

\section{Patient consent for publication}

Not applicable.

\section{Competing interests}

The authors declare that they have no competing interests.

\section{References}

1. Hirano T: Condensins: Universal organizers of chromosomes with diverse functions. Genes Dev 26: 1659-1678, 2012.

2. Wood AJ, Severson AF and Meyer BJ: Condensin and cohesin complexity: The expanding repertoire of functions. Nat Rev Genet 11: 391-404, 2010.

3. Schleiffer A, Kaitna S, Maurer-Stroh S, Glotzer M, Nasmyth K and Eisenhaber F: Kleisins: A superfamily of bacterial and eukaryotic SMC protein partners. Mol Cell 11: 571-575, 2003.

4. Zhang Q, Su R, Shan C, Gao C and Wu P: Non-SMC condensin i complex, subunit $\mathrm{G}$ (NCAPG) is a novel mitotic gene required for hepatocellular cancer cell proliferation and migration. Oncol Res 26: 269-276, 2018.

5. Yin L, Jiang LP, Shen QS, Xiong QX, Zhuo X, Zhang LL, Yu HJ, Guo X, Luo Y, Dong J, et al: NCAPH plays important roles in human colon cancer. Cell Death Dis 8: e2680, 2017.

6. Ryu B, Kim DS, Deluca AM and Alani RM: Comprehensive expression profiling of tumor cell lines identifies molecular signatures of melanoma progression. PLoS One 2: e594, 2007.

7. Liu W, Liang B, Liu H, Huang Y, Yin X, Zhou F, Yu X, Feng Q, $\mathrm{Li} \mathrm{E}, \mathrm{Zou} \mathrm{Z}$ and $\mathrm{Wu} \mathrm{L}$ : Overexpression of non-SMC condensin I complex subunit $\mathrm{G}$ serves as a promising prognostic marker and therapeutic target for hepatocellular carcinoma. Int J Mol Med 40: 731-738, 2017.

8. Robinson D, Van Allen EM, Wu YM, Schultz N, Lonigro RJ, Mosquera JM, Montgomery B, Taplin ME, Pritchard CC, Attard G, et al: Integrative clinical genomics of advanced prostate cancer. Cell 161: 1215-1228, 2015.

9. Chen C, Sun C, Tang D, Yang G, Zhou X and Wang D: Identification of key genes in glioblastoma-associated stromal cells using bioinformatics analysis. Oncol Lett 11: 3999-4007, 2016.

10. Nowroozi MR, Momeni SA, Ohadian Moghadam S, Ayati E, Mortazavi A, Arfae S, Jamshidian H, Taherimahmoudi M and Ayati M: Prostate-specific antigen density and gleason score predict adverse pathologic features in patients with clinically localized prostate cancer. Nephrourol Mon 8: e39984, 2016.

11. Kuriyama M, Wang MC, Lee CI, Papsidero LD, Killian CS, Inaji H, Slack NH, Nishiura T, Murphy GP and Chu TM: Use of human prostate-specific antigen in monitoring prostate cancer. Cancer Res 41: 3874-3876, 1981.

12. Bazett-Jones DP, Kimura K and Hirano T: Efficient supercoiling of DNA by a single condensin complex as revealed by electron spectroscopic imaging. Mol Cell 9: 1183-1190, 2002.

13. Neuwald AF and Hirano T: HEAT repeats associated with condensins, cohesins, and other complexes involved in chromosome-related functions. Genome Res 10: 1445-1452, 2000.

14. Viera A, Gomez R, Parra MT, Schmiesing JA, Yokomori K, Rufas JS and Suja JA: Condensin I reveals new insights on mouse meiotic chromosome structure and dynamics. PLoS One 2: e783, 2007.

15. Buyyounouski MK, Choyke PL, McKenney JK, Sartor O, Sandler HM, Amin MB, Kattan MW and Lin DW: Prostate cancer-major changes in the American joint committee on cancer eighth edition cancer staging manual. CA Cancer J Clin 67: 245-253, 2017.

16. Gao J, Aksoy BA, Dogrusoz U, Dresdner G, Gross B, Sumer SO, Sun Y, Jacobsen A, Sinha R, Larsson E, et al: Integrative analysis of complex cancer genomics and clinical profiles using the cBioPortal. Sci Signal 6: pl1, 2013.

17. Uhlen M, Zhang C, Lee S, Sjöstedt E, Fagerberg L, Bidkhori G, Benfeitas R, Arif M, Liu Z, Edfors F, et al: A pathology atlas of the human cancer transcriptome. Science 357: pii: eaan2507, 2017.

18. Taylor BS, Schultz N, Hieronymus H, Gopalan A, Xiao Y, Carver BS, Arora VK, Kaushik P, Cerami E, Reva B, et al: Integrative genomic profiling of human prostate cancer. Cancer Cell 18: 11-22, 2010.

19. Jia Z, Wang Y, Sawyers A, Yao H, Rahmatpanah F, Xia XQ, $\mathrm{Xu} \mathrm{Q}$, Pio R, Turan T, Koziol JA, et al: Diagnosis of prostate cancer using differentially expressed genes in stroma. Cancer Res 71: 2476-2487, 2011. 
20. Arredouani MS, Lu B, Bhasin M, Eljanne M, Yue W, Mosquera JM, Bubley GJ, Li V, Rubin MA, Libermann TA and Sanda MG: Identification of the transcription factor single-minded homologue 2 as a potential biomarker and immunotherapy target in prostate cancer. Clin Cancer Res 15: 5794-5802, 2009.

21. Dennis G Jr, Sherman BT, Hosack DA, Yang J, Gao W, Lane HC and Lempicki RA: DAVID: Database for annotation, visualization, and integrated discovery. Genome Biol 4: P3, 2003.

22. Jensen LJ, Kuhn M, Stark M, Chaffron S, Creevey C, Muller J, Doerks T, Julien P, Roth A, Simonovic M, et al: STRING 8-a global view on proteins and their functional interactions in 630 organisms. Nucleic Acids Res 37 (Database Issue): D412-D416, 2009.

23. Shannon P, Markiel A, Ozier O, Baliga NS, Wang JT, Ramage D, Amin N, Schwikowski B and Ideker T: Cytoscape: A software environment for integrated models of biomolecular interaction networks. Genome Res 13: 2498-2504, 2003.

24. Wang Y, Xia XQ, Jia Z, Sawyers A, Yao H, Wang-Rodriquez J, Mercola D and McClelland M: In silico estimates of tissue components in surgical samples based on expression profiling data. Cancer Res 70: 6448-6455, 2010.

25. Zhang Y, Li Z, Yang M, Wang D, Yu L, Guo C, Guo X and Lin N: Identification of GRB2 and GAB1 coexpression as an unfavorable prognostic factor for hepatocellular carcinoma by a combination of expression profile and network analysis. PLoS One 8: e85170, 2013.
26. Miao L, Huang Z, Zengli Z, Li H, Chen Q, Yao C, Cai H, Xiao Y, $\mathrm{Xia} \mathrm{H}$ and Wang Y: Loss of long noncoding RNA FOXF1-AS1 regulates epithelial-mesenchymal transition, stemness and metastasis of non-small cell lung cancer cells. Oncotarget 7: 68339-68349, 2016.

27. Vidal M, Cusick ME and Barabasi AL: Interactome networks and human disease. Cell 144: 986-998, 2011.

28. Huttlin EL, Bruckner RJ, Paulo JA, Cannon JR, Ting L, Baltier K, Colby G, Gebreab F, Gygi MP, Parzen H, et al: Architecture of the human interactome defines protein communities and disease networks. Nature 545: 505-509, 2017.

29. Tapon N, Harvey KF, Bell DW, Wahrer DC, Schiripo TA, Haber D and Hariharan IK: Salvador promotes both cell cycle exit and apoptosis in Drosophila and is mutated in human cancer cell lines. Cell 110: 467-478, 2002.

30. Hartwell L: Defects in a cell cycle checkpoint may be responsible for the genomic instability of cancer cells. Cell 71: 543-546, 1992. 